\title{
Neuromodulation et ontogenèse des réseaux de neurones moteurs
}

\section{$\mathbf{L}$} es activités rythmiques impliquées dans des comportements moteurs élémentaires, tels que respiration, locomotion, mastication, déglutition, sont essentielles à la survie de l'espèce. Au niveau du système nerveux central, ces activités sont mises en œuvre par des groupes de neurones interconnectés formant des réseaux distincts. De nombreux travaux ont permis de dégager les principes généraux du fonctionnement de ces réseaux [1]. Quelle que soit l'activité impliquée, ce fonctionnement repose d'une part sur les propriétés synaptiques qui lient les neurones entre eux et permettent de produire des séquences d'activités, et d'autre part sur les propriétés intrinsèques des éléments constitutifs de ces réseaux comme les propriétés oscillatoires des neurones permettant la genèse du rythme. La résultante de l'ensemble de ces propriétés permet de produire des séquences d'activités motrices rythmiques. De plus, l'efficacité des relations synaptiques ainsi que l'expression des propriétés intrinsèques des neurones ne sont pas invariantes et peuvent être modifiées par des informations modulatrices provenant soit de la périphérie, soit d'autres régions du système nerveux central, ce qui permet à ces réseaux d'exprimer un large répertoire d'activités motrices [1].

Si les principes d'organisation de ces réseaux chez l'adulte sont maintenant bien connus, leur mise en place et leur maturation au cours du développement restent encore très peu explorées. En effet, alors que de nombreux travaux se sont intéressés aux mécanismes cellulaires et moléculaires du développement précoce du système nerveux central (neuro- genèse [2], migration [3], connectivité [4] et développement des propriétés intrinsèques [5]) conduisant à la mise en place de réseaux immatures, peu d'études ont abordé le problème du passage de ces réseaux aux réseaux adultes. Ces processus font intervenir des mécanismes complexes s'étendant parfois sur plusieurs mois - c'est le cas par exemple des réseaux respiratoires ou locomoteurs chez l'homme - et tout dysfonctionnement dans cette phase critique de maturation peut se révéler fatal ou entraîner de graves déficits chez l'adulte.

Principes généraux de mise en place des réseaux moteurs au cours de l'ontogenèse

Il est classiquement admis qu'il existe trois principes de mise en place des réseaux moteurs rythmiques (pour revue, voir [6]). Premièrement, les futurs réseaux adultes sont présents très tôt au cours du développement, soit sous forme de réseaux silencieux qui seront ensuite activés par des informations modulatrices (réseau neuronal contrôlant le vol chez le criquet [7]), soit sous forme de réseaux immatures dont les propriétés synaptiques et intrinsèques seront progressivement modifiées (réseaux neuronaux contrôlant la locomotion chez les vertébrés [8]). Deuxièmement, les futurs réseaux adultes sont constitués à partir d'éléments d'anciens réseaux embıyonnaires. Par exemple, de larges réseaux embryonnaires métamériques subissent un phénomène de mort neuronale entraînant une réorganisation de leur structure (réseaux respiratoires des mammifères [9]); dans d'autres cas, ces réorganisations régressives sont accompagnées de l'ajout de nouveaux neurones par un phénomène de neurogenèse tardive (réseaux contrôlant les différents comportements moteurs chez les insectes avant et après la métamorphose, pour revue, voir [10]). Troisièmement, il a été montré plus récemment que plusieurs réseaux adultes peuvent émerger à partir d'une même population de neurones embryonnaires organisée en un réseau fonctionnel unique [11].

Quel que soit le mode de développement, il est implicitement admis que les réseaux adultes n'existent pas en tant que tels chez l'embryon et que leur mise en place se fait progressivement au cours du développement. Cependant, nous avons pu montrer récemment, en utilisant un modèle d'invertébré, que des réseaux de type adulte sont présents chez l'embryon très tôt au cours du développement, mais que leur expression est réprimée par des processus neuromodulateurs [12].

\section{Le modèle utilisé :}

le système nerveux stomatogastrique

L'étude des comportements rythmiques offre l'opportunité d'étudier des systèmes intégrés dont l'activité est prédictible, ce qui permet une approche expérimentale plus aisée. En ce qui concerne les activités motrices comme la locomotion, la respiration ou la prise de nourriture, les études les plus fécondes ont été réalisées sur des préparations d'invertébrés: insectes, crustacés, mollusques, annélides (pour revue, voir [13]). ('es animaux expriment en effet des comportements rythmiques 
complexes, mais ceux-ci proviennent d'un petit nombre de neurones bien identifiés que l'on peut caractériser morphologiquement et électrophysiologiquement d'une préparation à l'autre.

Un des modèles les mieux décrits est le système nerveux stomatogastrique des crustacés [14]. Ce système nerveux contrôle les mouvements rythmiques du tube digestif antérieur (œesophage et poche stomacale) lors de la prise de nourriture et de sa trituration (figure 1A). La poche stomacale des homards est constituée d'un moulinet gastrique comprenant trois puissantes dents qui broient les aliments (d'où le terme stomatogastrique), et d'une chambre pylorique qui contrôle le mouvement des fines particules alimentaires vers l'intestin moyen. Les mouvements rythmiques

Figure 1. Le système stomatogastrique des crustacés. A. L'estomac des crustacés constitue un lieu de transit, de stockage et de trituration des aliments ingérés par I'animal, grâce à une puissante musculature striée. Une partie du système nerveux, le système nerveux stomatogastrique, commande les mouvements rythmiques des différentes régions de l'estomac comme les dents du moulinet gastrique, mues par les muscles DG (dorsal gastric) et $G M$ (gastric mill), et la chambre pylorique, mue par les muscles LP (lateral pyloric) et $P D$ (pyloric dilatator). Les neurones ( $D G, G M, L P, P D)$ contrôlant ces muscles gastriques et pyloriques sont situés dans le ganglion stomatogastrique et reçoivent des informations modulatrices provenant de ganglions plus rostraux (ganglions antérieurs) via un unique nerf afférent, le nerf stomatogastrique (stn). L'activité produite par ces neurones est enregistrée par des techniques électrophysiologiques au niveau de leur soma. B. Chez l'embryon, l'activité des neurones du ganglion stomatogastrique peut être enregistrée intracellulairement au niveau de leurs cibles musculaires (muscles DG, GM, PD, LP), après ouverture et étalement de l'estomac embryonnaire. Comme chez l'adulte, le ganglion stomatogastrique reçoit des informations modulatrices en provenance des ganglions antérieurs via un unique nerf afférent (stn). de ces deux régions sont organisés par une musculature striée innervée par le système nerveux stomatogastrique (figure 1A). Ce système est constitué de quatre ganglions interconnectés dont le ganglion stomatogastrique qui contient 30 neurones. Chez l'adulte, cette population neuronale est organisée en deux réseaux, le réseau gastrique (16 neurones) et le réseau pylo- rique (14 neurones). Ce modèle possède une caractéristique remarquable: le système nerveux stomatogastrique, isolé dans une boîte de Pétri, produit spontanément et continuellement des activités rythmiques (figure 2A) semblables à celles qui sont enregistrées sur l'animal intact. Ces activités peuvent être enregistrées soit au niveau musculaire (figure $1 B$ ), soit

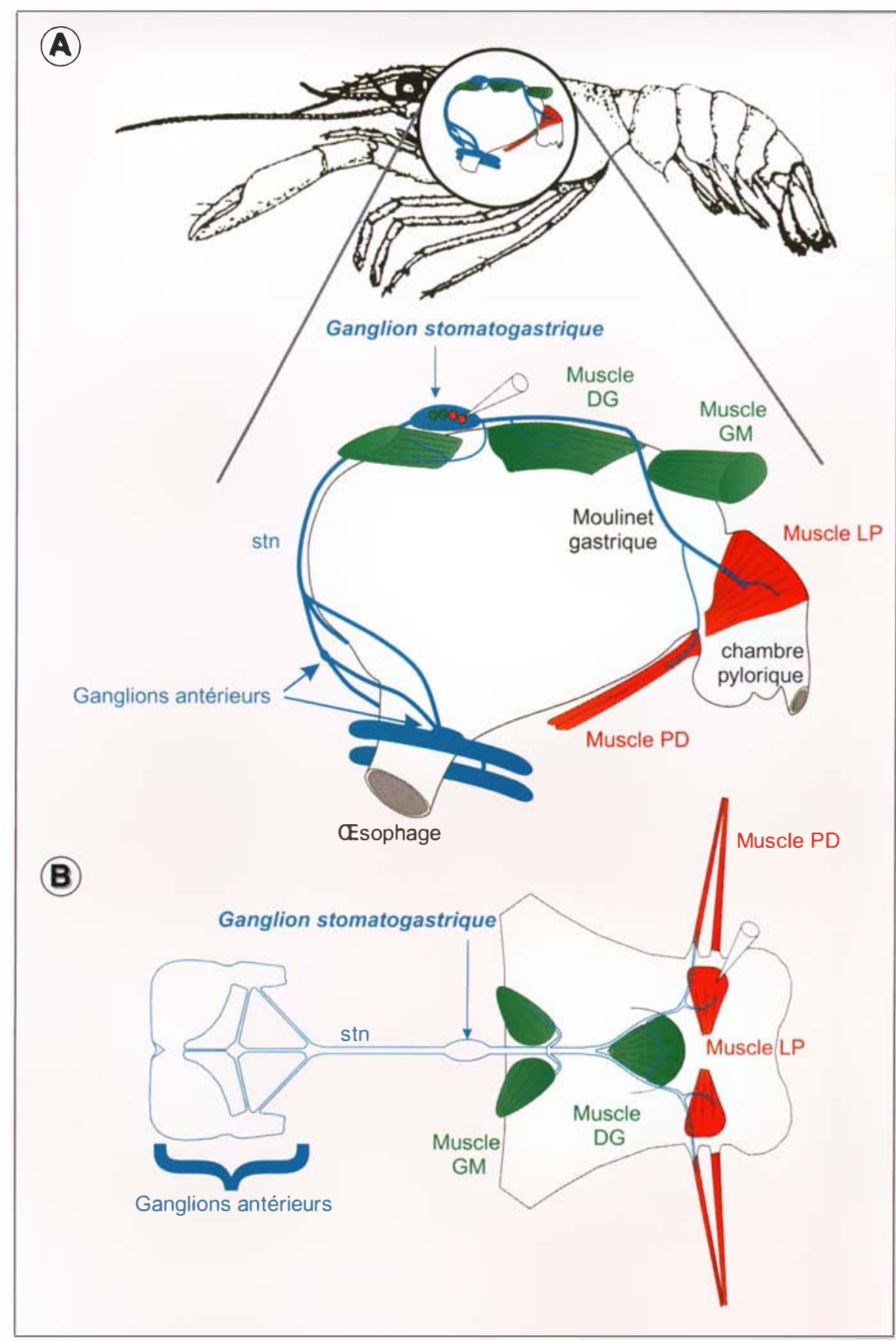




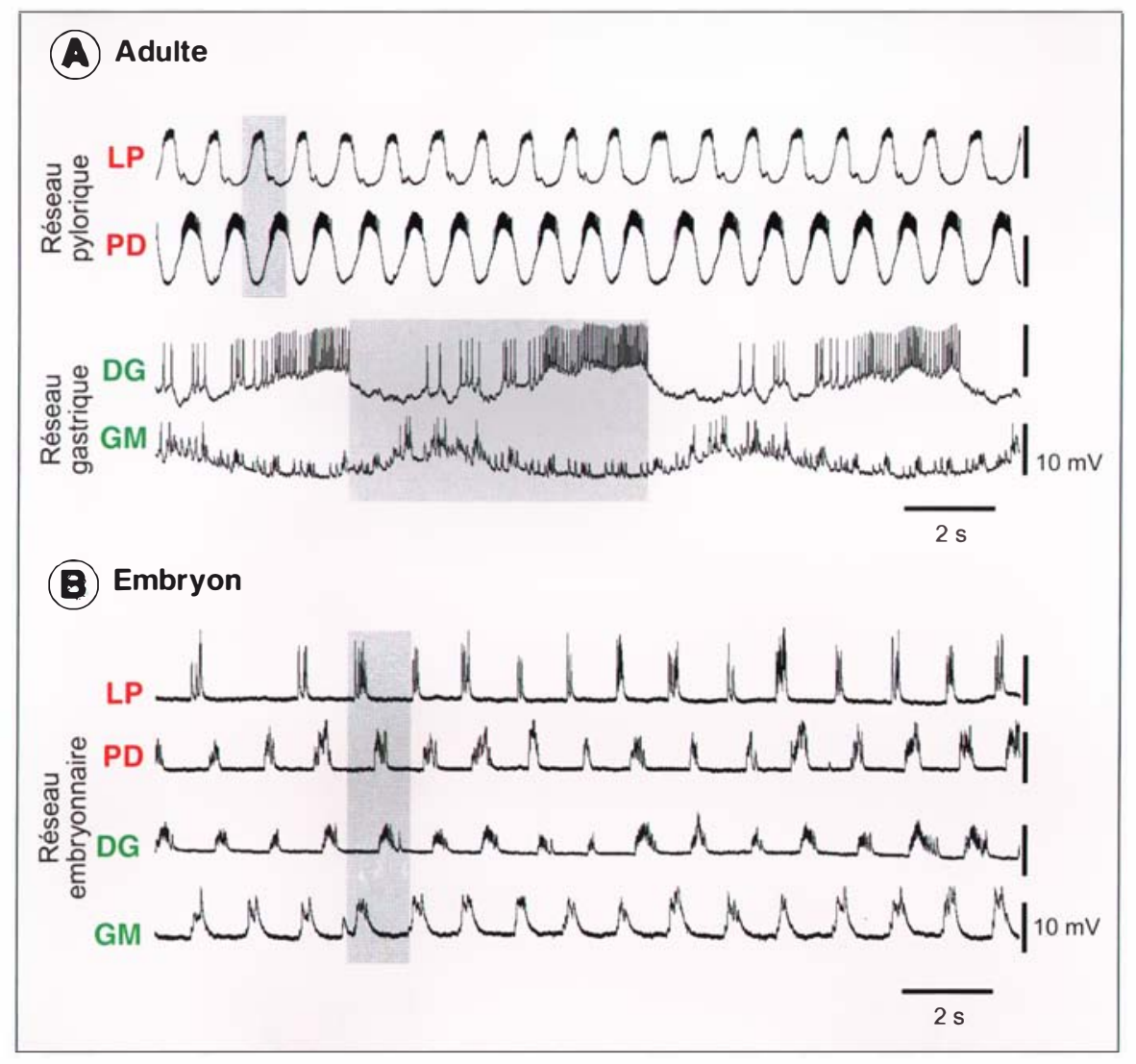

Figure 2. Activité motrice rythmique spontanée du système nerveux stomatogastrique adulte et embryonnaire chez le homard (Homarus gammarus). A. Chez l'adulte, les neurones du ganglion stomatogastrique sont organisés en deux réseaux moteurs distincts, les réseaux pylorique (LP et PD) et gastrique (DG et GM), qui produisent respectivement une activité rapide entraînant les muscles pyloriques et une activité lente entraînant les muscles gastriques. B. Chez l'embryon, les neurones du ganglion stomatogastrique sont organisés en un unique réseau moteur produisant spontanément une activité motrice rythmique unique, entraînant à la fois les muscles pyloriques et gastriques. Chaque réseau, pylorique, gastrique et embryonnaire, possède sa propre période soulignée par des zones grisées. (Figure adaptée à partir de [12].)

au niveau des motoneurones qui projettent sur ces muscles (figure 1A). Chez l'adulte, le ganglion stomatogastrique crée deux rythmes distincts: um rythme lent, le rythme gastrique, dont la période moyemne est d'environ 10 secondes, et un rythme plus rapide, le rythme pylorique, dont la période moyenne est d'environ 1 seconde (figure 2A). Le fonctionnement de ces deux réseaux repose d'une part sur les propriétés synaptiques (synapses électriques et synapses chimiques inhibitrices) et
Émergence de plusieurs réseaux adultes à partir d'un réseau embryonnaire unique

Le tube digestif antérieur et le système nerveux stomatogastrique sont identifiables très tôt au cours du développement (figure $1 B$ ), et le nombre de neurones du ganglion stomatogastrique est identique à celui de l'adulte quel que soit le stade de développement [15]. Ces neurones projettent sur les mêmes muscles que chez l'adulte, ce qui permet leur identification (jigure 1B). De plus, l'absence de division cellulaire au sein de cette population neuronale quand le développement embryonnaire a atteint $70 \%$ permet d'étudier les mêmes neurones tout au long du développement [15]. Chez l'embryon, cette population est organisée en un réseau unique qui produit spontanément une activité rythmique avec une période de $1,3 \mathrm{~s}$ (figure 2B), tandis que chez l'adulte, cette même population est organisée en deux réseaux, le réseau pylorique et le réseau gastrique (figure $2 A$ ).

Ces résultats démontrent que des réseaux fonctionnels adultes peuvent émerger à partir d'une population embryomnaire déjà existante et organisée en un unique réseau fonctionnel. L'étape suivante était donc de déterminer les mécanismes impliqués dans cette ségrégation ontogénique.

\section{Répression de l'expression}

des réseaux adultes par des afférences modulatrices chez l'embryon

Chez l'adulte, l'expression des réseaux stomatogastriques est strictement dépendante des voies modulatrices descendantes [14]. Nous avons donc émis l'hypothèse selon laquelle ces voies modulatrices pourraient jouer un rôle dans la maturation de ces réseaux embryommaires. Dans un premier temps, en utilisant des anticorps dirigés contre des neuromodulateurs, nous avons étudié leur chronologie d'apparition dans le ganglion stomatogastrique [15-17]. Avant $40 \%$ de développement embryønaire, aucun marquage n'est observé. Ce sont des neuropeptides 
qui sont d'abord détectés, à la moitié du développement [15], les substances aminergiques (sérotonine et dopamine) [16, 17] n'étant présentes que plus tardivement après la vie embryonnaire. Ces résultats montrent donc que l'acquisition des voies modulatrices au cours du développement est séquentielle. Cependant, malgré l'absence de corrélation évidente entre l'apparition de nouvelles voies modulatrices et la ségrégation des réseaux adultes, les afférences jouent un rôle primordial dans l'expression du réseau embryonnaire. En effet, comme chez l'adulte [14], le blocage expérimental des neurones modulateurs situés dans les ganglions antérieurs abolit l'activité du réseau embryonnaire (comparer figures $3 A$ et $3 B$ ). Chez l'adulte, la perfusion de substances modulatrices, directement sur les réseaux isolés de leurs afférences modulatrices, induit à nouveau leurs activités rythmiques. Parmi ces substances, les agonistes cholinergiques muscariniques sont capables de restaurer à la fois l'activité lente gastrique et l'activité rapide pylorique [14]. Chez l'embryon, dans les mêmes conditions expérimentales, ces agonistes sont eux aussi capables de déclencher des activités rythmiques. Cependant, au lieu de produire un rythme embryonnaire unique, cette stimulation pharmacologique amène la population neuronale embryonnaire à exprimer deux activités rythmiques distinctes (figure 3C). De façon remarquable, tous les neurones embryonnaires exprimant le rythme le plus lent deviendront des membres du réseau gastrique, tandis que tous ceux qui expriment le rythme le plus rapide appartiendront au réseau pylorique. De plus, dans de telles conditions expérimentales, les alternances entre les neurones antagonistes sont identiques à celles observées chez l'adulte [12].

Ces résultats indiquent que les caractéristiques des réseaux adultes peuvent être présentes très tôt au cours de la vie embryonnaire. Il apparaît de plus que leur activité est réprimée par des processus impliquant les voies modulatrices descendantes. En effet, si l'activité de ces voies modulatrices

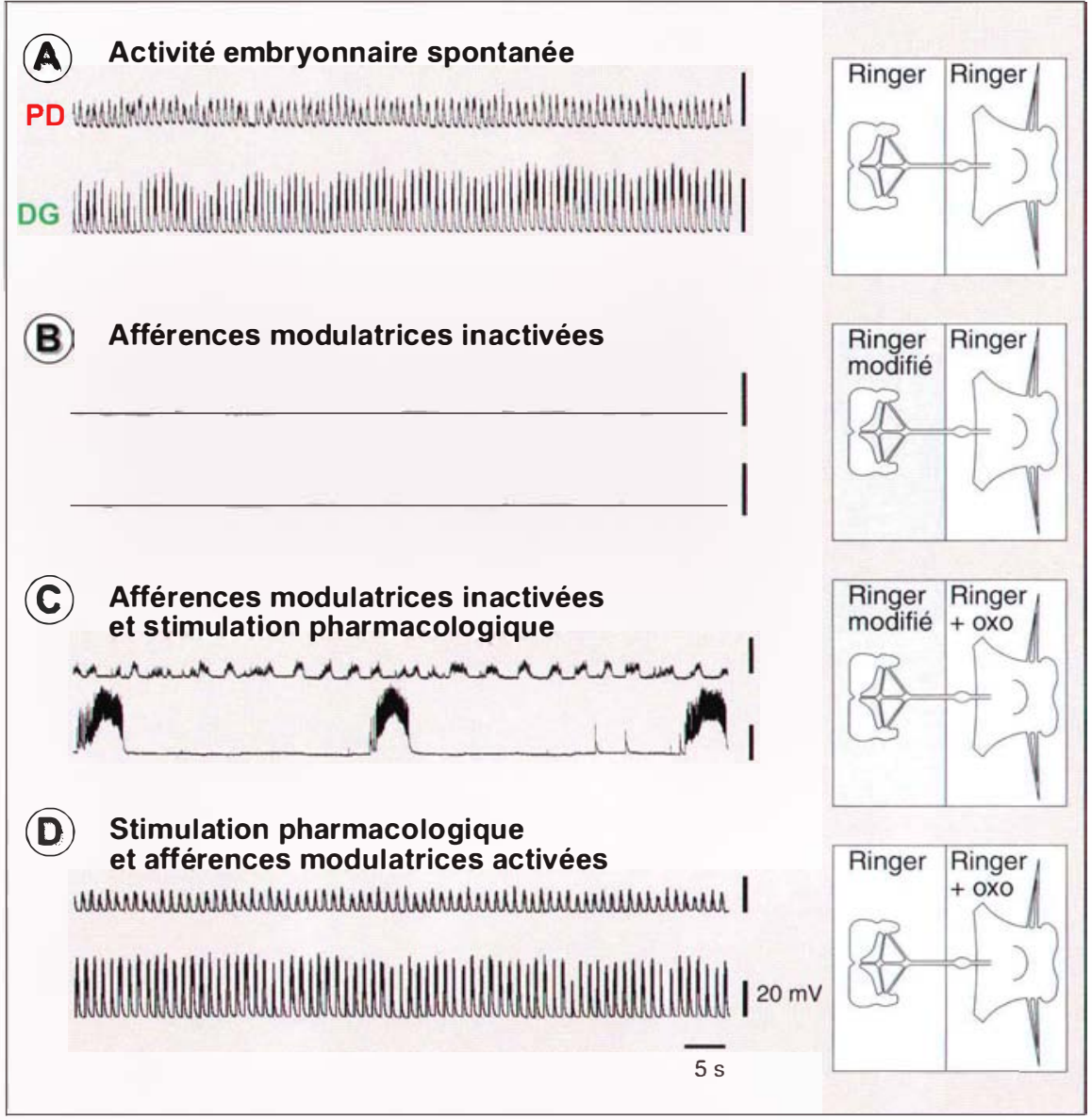

Figure 3. Les réseaux adultes sont présents chez l'embryon mais leur expression est réprimée par des afférences neuromodulatrices. A. Le système nerveux stomatogastrique embryonnaire isolé in vitro (schéma de droite) produit spontanément une activité motrice rythmique unique, enregistrée ici au niveau des cibles musculaires d'un neurone pylorique (PD) et $d^{\prime}$ un neurone gastrique (DG). B. Le blocage de l'activité des ganglions antérieurs (préparation décentralisée) par perfusion de Ringer modifié (sans sodium, schéma de droite), abolit l'activité des neurones du ganglion stomatogastrique embryonnaire. C. Sur une préparation décentralisée, l'application d'un agoniste cholinergique ayant des effets neuromodulateurs, l'oxotrémorine (oxo), induit l'expression de deux activités rythmiques distinctes ayant des caractéristiques adultes. Le rythme le plus lent est exprimé par la souspopulation de neurones gastriques (ici DG) tandis que le rythme le plus rapide est produit par des neurones pyloriques (ici PD). D. Dans les mêmes conditions expérimentales qu'en $C$, la restauration de l'activité des ganglions antérieurs (par rinçage, schéma de droite) provoque le retour au rythme embryonnaire unique. (Figure adaptée à partir de [12].)

cibles sont toujours stimulés pharmacologiquement, l'activité de type adulte disparaît pour laisser place à une activité embryonnaire typique (figure 3D). Ces résultats démontrent clairement que, chez l'embryon, l'activité des réseaux neuronaux adultes est réprimée par l'activité des voies modulatrices descendantes.

\section{Conclusions}

Alors que les mécanismes cellulaires qui sous-tendent l'organisation et la neuromodulation des réseaux moteurs commencent à être connus aussi bien chez les invertébrés que chez les vertébrés, ceux qui sont impliqués dans l'ontogenèse de ces 
réseaux restent méconnus. Néanmoins, un certain nombre de principes d'organisation commencent à émerger et l'implication des substances neuromodulatrices dans cette ontogenèse commence à être mieux définie.

I.es afférences neuromodulatrices sont ubiquitaires dans le système nerveux central et sont impliquées dans toutes les fonctions cérébrales. Elles jouent un rôle dans l'expression de réseaux de neurones, dans le contrôle de leur flexibilité, permettant une adaptation rapide aux modifications de l'environnement [1], et dans le maintien à long terme des caractéristiques du réseau [18]. Leur dysfonctionnement est responsable de nombreuses pathologies aussi bien sur le système moteur (maladie de Parkinson) que sur d'autres fonctions (anxiété, mémoire). Il est clair qu'elles jouent aussi un rôle primordial dans la mise en place et la maturation des réseaux de neurones (pour revue, voir [19]).

Une caractéristique commune à tous les réseaux de neurones moteurs est leur apparition très précoce au cours du développement. Cependant, les voies ontogéniques pour accéder au phénotype adulte sont variées (pour revue, voir [6]) : les réseaux précoces peuvent être maintenus silencieux jusqu'à ce que leur fonction deviemme nécessaire (le réseau du vol chez les insectes), ou bien acquérir progressivement les caractères adultes (le réseau de la nage chez les batraciens), ou encore être réorganisés par élimination de sousensembles neuronaux (le réseau respiratoire des mammifères) ou par ségrégation en réseaux distincts à partir d'un large réseau embryon-

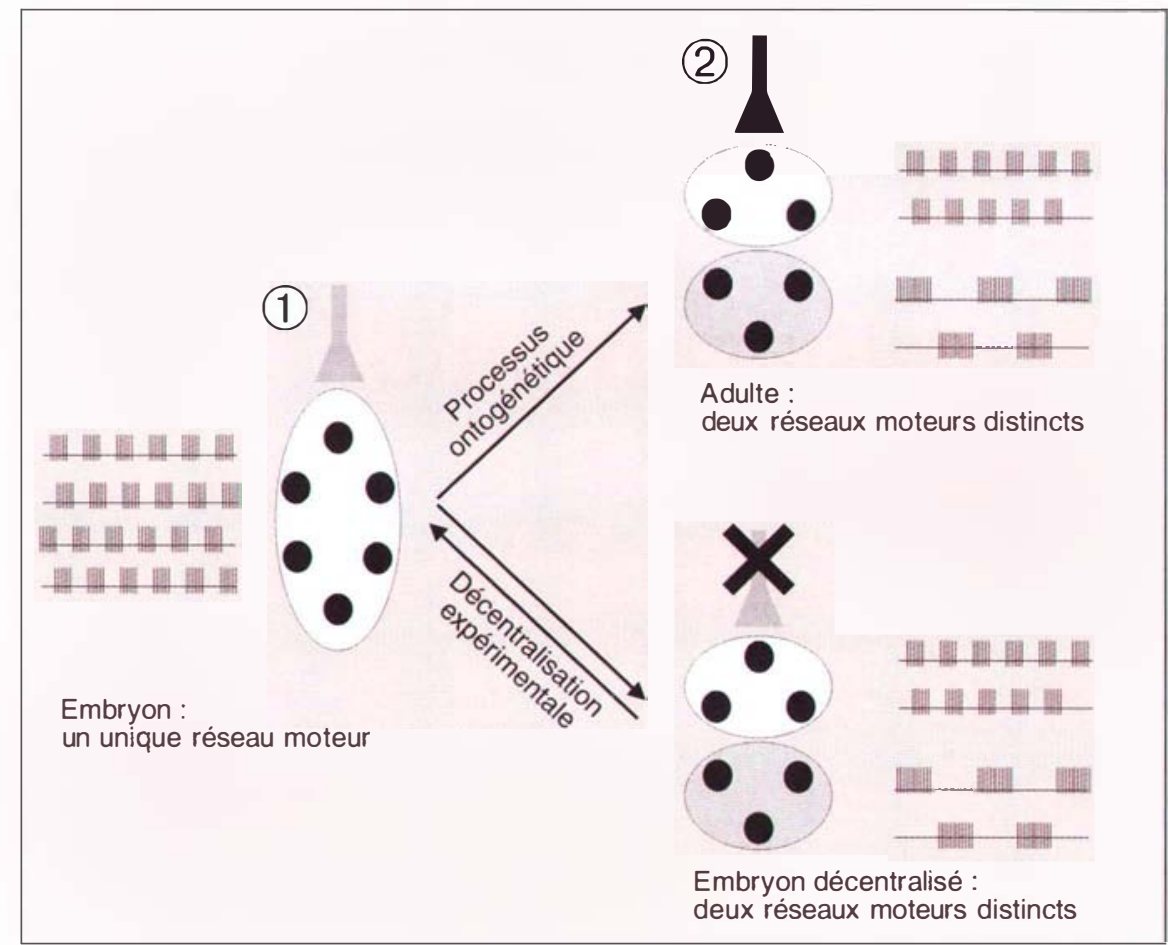

Figure 4. Ontogenèse des réseaux de neurones: répression des phénotypes adultes par des voies modulatrices centrales. Chez l'embryon, comme chez l'adulte, l'expression des réseaux dépend d'afférences modulatrices qui, bien qu'apparaissant précocement (1), acquièrent leurs caractéristiques adultes plus tardivement (2). Chez l'embryon, l'activité des neurones modulateurs maintient la population neuronale du ganglion stomatogastrique dans un unique réseau moteur fonctionnel. Chez l'adulte, la même population stomatogastrique est organisée en deux réseaux distincts. II est possible de mimer l'effet des processus ontogéniques aboutissant à la formation des deux réseaux adultes distincts en décentralisant la préparation embryonnaire.

$m / s{ }^{\circ} 6-7$, vol. 16, juin-juillet 2000 naire (le réseau stomatogastrique des crustacés). Nos résultats montrent que des réseaux neuronaux adultes sont présents chez l'embryon mais que des voies modulatrices descendantes répriment leur expression, tout en maintenant ces neurones au sein d'un réseau embıyonnaire fonctiommel [12]. Ce principe de développement contraste avec les mécanismes ontogéniques déjà décrits dans lesquels les réseaux de neurones acquièrent progressivement leurs propriétés adultes au cours du développement.

En conclusion, les réseaux embryonnaires ne doivent pas être nécessairement vus comme des entités immatures mais peuvent également être considérés comme des réseaux de type adulte dont l'expression est réprimée, chez l'embryon, par un ensemble d'informations modulatrices (figure 4)

\section{RÉFÉRENCES}

1. Marder E, Calabrese RL. Principles of rhythmic motor pattern generation. Physiol Rev 1996; 76: 687-717.

2. Guillemot F. Signaux extracellulaires et programmes transcriptionnels contrôlant la neurogenèse. Med Sci 2000; 16: 159-63.

3. Hatten ME. Central nervous system neuronal migration. Annu Rev Neurosci 1999; 22: $511-39$.

4. Lee SH, Sheng M. Development of neuron-neuron synapses. Curr Opin Neurobiol $2000 ; 10: 125-31$.

5. Spitzer NC, Ribera AB. Development of electrical excitability in embryonic neurons : mechanisms and roles. J Neurobiol 1998; 37 : $190-7$.

6. Fénelon, VS, Casasnovas B, Simmers J, Meyrand P. Development of rhythmic pattern generators. Curr Opin Neurobiol 1998 ; 8: $705-9$.

7. Stevenson PA, Kutsch W. Demonstration of functional connectivity of the flight motor system in all stages of the locust. $J$ Comp Physiol A 1988; 162: 247-59.

8. Bate M. Development of motor behaviour. Curr Opin Neurobiol 1999; 9: 670-5.

9. Champagnat J, Fortin G. Primordial respiratory-like rhythm generation in the vertebrate embryo. Trends Neurosci $1997 ; 20: 119$ 24. 


\section{RÉFÉRENCES}

10. Truman JW. Developmental neuroethology of insect metamorphosis. I Neurobiol $1992 ; 23: 1404-22$.

11. Casasnovas B, Meyrand P. Functional differentiation of multiple neural circuits from a single embryonic network. $J$ Neurosci $1995 ; 15$ : 5703-18.

12. Le Feuvre Y, Fénelon VS, Meyrand $P$. Removal of central inputs unmasks multiple adult neural networks from a single embryonic network. Nature 1999; 402 : 660-4.

13. Calabrese RL, De Schutter E. Motor-pattern-generating networks in invertebrates: modeling our way toward understanding. Trends Neurosci $1992 ; 15: 439-45$.

14. Harris-Warrick RM, Marder E, Selverston AI, Moulins M. Dynamic biological net works The stomatogastric nervous system. Cam bridge. The MIT Press, 1992: 328
15. Fénelon VS, Casasnovas B, Faumont $S$, Meyrand P. Ontogenetic alteration in peptidergic expression within a stable neuronal population in lobster stomatogastric nervous system. J Comp Neurol 1998; 399: 289305 .

16. Kilman V, Fénelon VS, Richards KS, Thirumalai V, Meyrand $P$, Marder E. Sequential developmental acquisition of cotransmitters in identified neurons of the stomatogastric nervous system of the lobsters, Homarus americanus and Homarus gammarus. J Comp Neurol 1999; 408: 318-34.

17. Fénelon VS, Kilman V, Meyrand P, Marder E. Sequential developmental acquisition of neuromodulatory inputs to a central pattern generating network. I Comp Neurol 1999 ; 408: 335-51

18. Thoby-Brisson M, Simmers JA. Neuromodulatory inputs maintain expression of a lobster motor pattern-generating network in a modulation-dependent state: evidence from long-term decentralization in vitro. I Neurosci 1998; 18: 2212-25.

19. Pflüger H-J. Neuromodulation during motor development and behavior. Curr Opin Neurobiol 1999; 9 : 683-9.

\section{Valérie S. Fénelon}

Yves Le Feuvre

Pierre Meyrand

Laboratoire de neurobiologie des réseaux, Cnrs, Université Bordeaux I, UMR 5816 , avenue des Facultés, 33405 Talence Cedex, France.

\section{TIRÉS À PART}

P. Meyrand.

\section{$C 1 \Rightarrow$ centrenational DE LA RECHERCHE SCIENTIFIQUE}

du 11 au 15 septembre 2000 à MARSEILLE (13)

du 2 au 5 octobre 2000 à MARSEILLE (13)

du 16 au 20 octobre 2000

à ORSAY $(91)$

du 23 au 27 octobre 2000

à ORSAY (91)

du 20 au 21 novembre 2000

à VENDOME $(41)$

du 20 au 24 novembre 2000

à ORSAY (91)

\section{CNRSFormation \\ au service de l'Entreprise}

Connaissance et protection de l'animal de laboratoire : entretien et soins. Niveau III

Connaissance de l'animal de laboratoire : méthodologie expérimentale. Spécialisation chirurgie niveau I

Méthodes d'analyse et stratégies d'identification des protéines pour techniciens

Méthodes d'analyse et stratégies d'identification des protéines pour chercheurs et ingénieurs

Module complémentaire des stages d'habilitation Lagomorphes : lapins (Enseignement pratique)

La PCR : quelques applications 